\title{
Estimating the parameters of globular cluster M 30 (NGC 7099) from time-series photometry (Corrigendum)
}

N. Kains ${ }^{1}$, D. M. Bramich ${ }^{1}$, A. Arellano Ferro ${ }^{2}$, R. Figuera Jaimes ${ }^{1,3}$, U. G. Jørgensen ${ }^{4,5}$, S. Giridhar ${ }^{6}$, M. T. Penny ${ }^{7}$, K. A. Alsubai ${ }^{8}$, J. M. Andersen ${ }^{9,5}$, V. Bozza ${ }^{10,11}$, P. Browne ${ }^{3}$, M. Burgdorf ${ }^{12}$, S. Calchi Novati ${ }^{10,13}$, Y. Damerdji ${ }^{14}$, C. Diehl ${ }^{15,16}$, P. Dodds ${ }^{3}$, M. Dominik ${ }^{3, \star}$, A. Elyiv ${ }^{14,17}$, X.-S. Fang ${ }^{18,19}$, E. Giannini ${ }^{15}$, S.-H. Gu ${ }^{18,19}$, S. Hardis ${ }^{4}$, K. Harpsøe $\mathrm{e}^{4,5}$, T. C. Hinse ${ }^{20,4}$, A. Hornstrup ${ }^{21}$, M. Hundertmark ${ }^{3}$, J. Jessen-Hansen ${ }^{22}$, D. Juncher ${ }^{4,5}$, E. Kerins ${ }^{23}$, H. Kjeldsen ${ }^{22}$, H. Korhonen ${ }^{4,5}$, C. Liebig ${ }^{3}$, M. N. Lund ${ }^{22}$, M. Lundkvist ${ }^{22}$, L. Mancini' ${ }^{24}$, R. Martin ${ }^{25}$, M. Mathiasen ${ }^{4}$, M. Rabus ${ }^{26}$, S. Rahvar ${ }^{27,28}$, D. Ricci ${ }^{29,14}$, K. Sahu ${ }^{30}$, G. Scarpetta ${ }^{10,31}$, J. Skottfelt ${ }^{4,5}$, C. Snodgrass ${ }^{32}$, J. Southworth ${ }^{33}$, J. Surdej ${ }^{14}$, J. Tregloan-Reed ${ }^{33}$, C. Vilela ${ }^{33}$, O. Wertz ${ }^{14}$, and A. Williams ${ }^{25}$

(The MiNDSTEp consortium)

(Affiliations can be found after the references)

A\&A 555, A36 (2013), DOI: 10.1051/0004-6361/201321819

Key words. stars: variables: general - stars: variables: RR Lyrae - globular clusters: individual: M 30 (NGC 7099) - errata, addenda

The paper "Estimating the parameters of globular cluster M 30 (NGC 7099) from time-series photometry", used an incorrect expression for the phase $\phi(t)$ at time $t$ of a periodic variable undergoing a linear period change. Equation (3) of that paper should be:

$\phi(t)=N_{E}(t)-\left\lfloor N_{E}(t)\right\rfloor$,

where $N_{E}(t)$ is the number of cycles elapsed at time $t$ since a reference epoch $E$. For a constant rate of period change $\beta, N_{E}(t)$ is given by (Kains et al. 2015):

$N_{E}(t)=\frac{1}{\beta} \ln \left[1+\frac{\beta}{P_{0}}(t-E)\right]$,

where $P_{0}$ is the period at the epoch $E$. Using this correct expression, we are still unable to phase the light curve of RR Lyrae star V16 satisfactorily, and our conclusions therefore remain unaffected.

\section{References}

Kains, N., Arellano Ferro, A., Figuera Jaimes, R., et al. 2015, A\&A, 578, A128

1 European Southern Observatory, Karl-Schwarzschild Straße 2, 85748 Garching bei München, Germany

e-mail: nkains@eso.org

2 Instituto de Astronomía, Universidad Nacional Autónoma de Mexico 0410 Ciudad de México, Mexique

SUPA School of Physics \& Astronomy, University of St. Andrews, North Haugh, St. Andrews, KY16 9SS, UK

${ }^{4}$ Niels Bohr Institute, University of Copenhagen, Juliane Maries vej 30, 2100 Copenhagen, Denmark

5 Centre for Star and Planet Formation, Geological Museum, Øster Voldgade 5, 1350 Copenhagen, Denmark

Indian Institute of Astrophysics, 560034 Koramangala, Bangalore, India

7 Department of Astronomy, Ohio State University, 140 West 18th Avenue, Columbus, OH 43210, USA

${ }^{8}$ Qatar Foundation, PO Box 5825, Doha, Qatar

\footnotetext{
* Royal Society University Research Fellow.
}

9 Department of Astronomy, Boston University, 725 Commonwealth Ave, Boston, MA 02215, USA

10 Dipartimento di Fisica "E.R Caianiello", UniversitĹ di Salerno,

via Ponte Don Melillo, 84084 Fisciano, Italy

11 Istituto Nazionale di Fisica Nucleare, Sezione di Napoli, 80100 Napoli, Italy

12 HE Space Operations GmbH, Flughafenallee 24, 28199 Bremen, Germany

3 Istituto Internazionale per gli Alti Studi Scientifici (IIASS), 84019 Vietri Sul Mare (SA), Italy

14 Institut d'Astrophysique et de Géophysique, Université de Liège, Allée du 6 Août 17, Sart Tilman, Bât. B5c, 4000 Liège, Belgium

15 Astronomisches Rechen-Institut, Zentrum für Astronomie der Universität Heidelberg (ZAH), Mönchhofstr. 12-14, 69120 Heidelberg, Germany

16 Hamburger Sternwarte, Universität Hamburg, Gojenbergsweg 112, 21029 Hamburg, Germany

17 Main Astronomical Observatory, Academy of Sciences of Ukraine, vul. Akademika Zabolotnoho 27, 03680 Kyiv, Ukraine

18 National Astronomical Observatories/Yunnan Observatory, Chinese Academy of Sciences, 650011 Kunming, PR China

19 Key Laboratory for the Structure and Evolution of Celestial Objects, Chinese Academy of Sciences, 650011 Kunming, PR China

20 Korea Astronomy and Space Science Institute, 305-348 Daejeon, Korea

21 Institut for Rumforskning og -teknologi, Danmarks Tekniske Universitet, 2800 København, Denmark

22 Stellar Astrophysics Centre, Department of Physics and Astronomy, Aarhus University, Ny Munkegade 120, 8000 Aarhus C, Denmark

23 Jodrell Bank Centre for Astrophysics, University of Manchester, Oxford Road,Manchester, M13 9PL, UK

24 Max Planck Institute for Astronomy, Königstuhl 17, 69117 Heidelberg, Germany

25 Perth Observatory, Walnut Road, Bickley, 6076 Perth, Australia

26 Departamento de Astronomía y Astrofísica, Pontificia Universidad Católica de Chile, Av. Vicuña Mackenna 4860, 7820436 Macul, Santiago, Chile

27 Department of Physics, Sharif University of Technology, PO Box 4, 11155-9161 Tehran, Iran

28 Perimeter Institute for Theoretical Physics, 31 Caroline St. N., Waterloo ON, N2L 2Y5, Canada

29 Instituto de Astronomía - UNAM, Km 103 Carretera Tijuana Ensenada, 422860 Ensenada (Baja Cfa), Mexico

30 Space Telescope Science Institute, 3700 San Martin Drive, Baltimore, MD 21218, USA

31 INFN, Gruppo Collegato di Salerno, Sezione di Napoli, Italy

32 Max Planck Institute for Solar System Research, Max-Planck-Str. 2, 37191 Katlenburg-Lindau, Germany

33 Astrophysics Group, Keele University, Staffordshire, ST5 5BG, UK 